\title{
Deploying high-throughput protein crystallography-based drug discovery platforms to establish a structure-based drug discovery system for SARS-CoV-2 proteins D Das ${ }^{1}$ \\ ${ }^{1}$ Accelero Biostructures \\ info@accelerobio.com
}

The novel SARS coronavirus (SARS-CoV-2) global pandemic has taken a strong foothold. There is a strong need for effective treatments that can be administered to infected individuals. We are helping to adopt a structure-based drug discovery approach for the discovery and development of novel inhibitors of the SARS-CoV-2 viral spike protein $\mathrm{S} 1$ subunit (attachment inhibitors), and inhibitors of the viral spike protein S2 subunit (fusion inhibitors). Our goal is to target conserved amino acid residues within these proteins to discover and advance molecules that may inhibit them. These molecules could serve as pan-coronavirus inhibitors and broad-spectrum antiviral therapeutics against multiple coronavirus strains and against the homologous proteins in SARS and MERS, thereby effectively creating treatments for both current and future coronavirus outbreaks. Coronaviruses (CoVs) infect humans and animals and cause a variety of diseases, primarily respiratory. There are estimated to be hundreds to thousands of CoVs in bats and other species and there have been at least three documented transmissions from animals to humans in the past 20 years leading to human viral pandemics and epidemics like COVID-19, SARS and MERS. It is only a matter of time before the next $\mathrm{CoV}$ hits us.

Over the past 20 years, primarily driven by high-throughput structural genomics and structural biology efforts in the USA and around the world, we have been involved in the development of instrumentation and automation for highthroughput protein X-ray crystallography, covering all stages of the gene-to-structure process. Outcomes include methods, processes, protocols, and instrumentation for high-throughput protein expression, purification, crystallization, structure determination, refinement, and analysis. Benefits have now percolated into industry and academia, influencing both structural biology and structure-based drug discovery research and have led to significant gains in efficiency and productivity at reduced cost. In addition, we have now built and deployed our novel high-throughput crystallography-based drug discovery platforms, including screening molecular compound libraries, such as fragment and scaffold libraries directly by X-ray crystallography for hit generation, which provides a direct experimental route to high quality, high reliability and high value results. Our goal is establishing a structure-based drug discovery system for the discovery and development of novel SARS-COV-2 therapeutics.

Towards this effort, we have completed determination of the highest resolution experimental X-ray crystal structures of the apo proteins of SARS-CoV-2 (a) S1 RBD (human ACE2 Receptor Binding Domain) at 1.8 $\AA$ resolution; and (b) the S2 post-fusion core at $2.2 \AA$ resolution. In addition, we have also set up a biophysical assay for the S1 RBD using surface plasmon resonance. Our work resulting in high resolution experimental apo protein structures sets the stage for a target-focused structure-and biophysics-guided system for testing and validating compounds against SARS-CoV-2 S1 RBD and S2. 